DOI: 10.33947/2316-7394-V9N1-3527

\title{
DESENVOLVIMENTO DE APLICATIVO DE INTELIGÊNCIA ARTIFICIAL PARA ESTÍMULO E APRENDIZAGEM DE AUTISTAS PARA MELHORA NA COMUNICAÇÃO: ESTUDO DE CASO APAE ARUJÁ E POÁ
}

\section{DEVELOPMENT OF ARTIFICIAL INTELLIGENCE APPLICATION FOR STIMULATION AND APPEARANCE OF AUTISTS TO IMPROVE COMMUNICATION: CASE STUDY APAE ARUJÁ AND POÁ}

\author{
Bruno Roque de Lima ${ }^{1}$
}

\section{RESUMO}

O artigo objetiva-se desenvolver uma aplicação de ensino para dispositivos móveis com foco em crianças com os distúrbios como Síndrome de Asperger, transtorno Desintegrativo e transtorno Generalizado, que unidos classificam-se como um único diagnóstico chamando de Transtorno do Espectro Autista (TEA), que é uma condição de desordens complexas no desenvolvimento do cérebro, que afeta antes, durante e logo após o nascimento do bebê, caracterizando a dificuldade na comunicação, coordenação motora, aprendizagem e atenção. Sendo assim o objetivo é auxiliar e melhorar o método de ensino de escolas e professores, implementando uma metodologia de exercícios e materiais didáticos, acompanhado de medição da evolução no desenvolvimento das atividades de coordenação motora, leitura, escrita e fala que serão passadas diariamente para os alunos de maneira educacional e interativa que auxiliará no desenvolvimento das suas maiores dificuldades.

PALAVRAS-CHAVE: Autismo. Sistema. Ensino. Desenvolvimento. Educação.

\begin{abstract}
The aim of this article is to develop a teaching application for mobile devices focused on children with disorders such as Asperger's Syndrome, Disintegrative Disorder and Generalized Disorder, which are classified as a single diagnosis called Autism Spectrum Disorder (ASD) which is a condition of complex disorders in the development of the brain, which affects before, during and soon after the birth of the baby, characterizing the difficulty in communication, motor coordination, learning and attention. Therefore, the objective is to assist and improve the teaching method of schools and teachers, implementing a methodology of exercises and didactic materials, accompanied by measurement of the evolution in the development of activities of motor coordination, reading, writing and speaking, which will be passed daily to the students in an educational and interactive way that will aid in the development of their greatest difficulties.
\end{abstract}

KEYWORDS: Autism. System. Teaching. Development. Education.

\footnotetext{
${ }^{1}$ Graduado em Ciência da Computação - 8 semestre. UNG - Universidade Guarulhos.brunogamovo@gmail.com.
} 


\section{Computa gevsta Rplicada}

\section{INTRODUÇÃO}

Autismo é um tema intrigante devido às incertezas de sua causa e aos questionamentos relacionados a ele que não possuem uma resposta exata.

O termo autista surgiu na literatura psiquiátrica com Plouller em 1906, ao estudar pacientes que tinham o diagnóstico de demência precoce, mas foi Eugen Bleuer, psiquiatra suíço, em 1911, o primeiro a difundir o termo autismo definindo-o "como perda de contato com a realidade, causada pela impossibilidade ou grande dificuldade na interação interpessoal" (SALLE et al., 2002, p. 11).

O autismo é uma condição permanente, a criança nasce com autismo e torna-se um adulto com autismo. Assim, essas diferenças podem existir desde o nascimento e serem óbvias para todos ou podem ser mais sutis e tornarem-se mais visíveis ao longo do desenvolvimento.

A partir do último Manual de Saúde Mental - DSM5 , algumas pessoas com TEA podem ter dificuldades de aprendizagem em diversos estágios da vida, desde estudar na escola, até aprender atividades da vida diária. Essas dificuldades fazem com que o aluno com autismo tenha certas limitações no processo de ensino-aprendizagem. Os principais e mais reconhecidos comprometimentos do transtorno autista encontram-se nas áreas da comunicação, comportamento e interação social. Apesar da dificuldade de comprometimentos de um aluno com autismo, sua escolarização é possível quando the são oferecidas oportunidades para que esse processo ocorra, como um professor que tenha conhecimento sobre os aspectos do autismo e que tenha conhecimento sobre os programas e métodos educacionais mais adequados para ensiná-lo.

Embora se discuta muito sobre o processo de inclusão do aluno com deficiência, ainda há um grande contingente de alunos que frequentam apenas a escola de educação especial, como o caso de alunos com autismo. Verificando essa situação, decidiu-se observar e analisar como se dá o processo de escolarização dos alunos com autismo em uma escola de educação especial, identificando a rotina diária da sala de aula e principalmente a metodologia empregada pelo professor para trabalhar com esse aluno.

Nilsson (2003) afirma que é preciso usar algumas estratégias visuais, como: Programação diária individual, um sistema de trabalho individual, atividades adaptadas individualmente para o trabalho independente, obrigações diárias apresentadas visualmente, atividades recreativas e atividades motoras, dando suporte adicional com a orientação visual do modo como a sala é mobiliada e usada.

Já existem alguns aplicativos móveis que introduzem uma forma de comunicação prática no relacionamento com famílias e pessoas da comunidade por meio de símbolos e imagens especificas através dos telefones celulares, eles formam palavras simples e frases ou até mesmo algumas figuras para expressar seus sentimentos e necessidades.

Nesta perspectiva é proposto no presente artigo um ambiente para dar suporte e auxiliar na educação de crianças com autismo através de diversas atividades. As atividades presentes no software foram desenvolvidas considerando aspectos importantes para estimular a concentração, o foco e o raciocínio logico das crianças utilizando-se das ferramentas de Inteligência Artificial que se propõe a elaborar dispositivos que simulem a capacidade humana de raciocinar, perceber, tomar decisões e resolver problemas e Machine Learning para ser um sistema inteligente, com capacidade de aprender com o histórico de dados. Assim diferenciando este software dos demais disponíveis através de pesquisas e entrevistas com profissionais das áreas de Psicologia e afins, objetivando ainda prover uma forma de mensurar a evolução da criança para diferentes tipos de atividades a partir de dados coletados à medida que as crianças utilizam os jogos, podendo esse ajudar os usuários reconhecendo as suas emoções através das suas reações diante do aplicativo independente dos diferentes tipos de perfis, podendo o software aprender e se adaptar de acordo com a autonomia do autista, tendo domínio sobre o conjunto de símbolos para que ele consiga utilizá-lo no processo comunicativo, no qual devem fazer sentido ao autista. Dessa forma, o software aprende com os movimentos realizados e se adapta às necessidades e personalidades de cada um dos usuários, o que facilita na aproximação e no interesse do autista criando até mesmo uma inclusão social.

\section{MATERIAIS E MÉTODOS}

Como o ambiente é um projeto dinâmico por aprender e conhecer cada usuário, a sua implantação dentro da escola vai atender as necessidades dos diferentes graus de autismos presentes. Assim possibilitando inclusão e estímulo dos alunos, podendo auxiliar os professores no acompanhamento das atividades passadas 


\section{Computa gevsta Rplicada}

diariamente e também no desenvolvimento das atividades de coordenação motora, leitura, escrita e fala, visando reforçar a evolução das dificuldades comuns que são a organização, distração e dificuldade em sequenciar.

O projeto consiste em um aplicativo para dispositivos móveis com ênfase em tablets e tem como recurso o uso de Inteligência Artificial, Machine Learning, Banco de Dados e um API REST e possui uma página web para a parte administrativa do sistema onde se gerencia os professores e alunos.

Um sistema de Inteligência Artificial deve ser capaz de fazer três coisas: armazenar conhecimento, aplicar o conhecimento armazenado para resolver problemas e adquirir novo conhecimento através da experiência, ou seja, representação, raciocínio e aprendizagem.

Segundo (Korth, 2004), um banco de dados é uma coleção de dados inter-relacionados, representando informações sobre um domínio específico, ou seja, sempre que for possível agrupar informações que se relacionam e tratam de um mesmo assunto, posso dizer que tenho um banco de dados.

(Arthur, 1959) afirma que Machine Learning é um campo de estudo que dá à computadores a capacidade de aprender sem ser programado de forma explícita.

Com isso, para a comunicação entre os sistemas é realizada a alimentação do banco de dados, onde um serviço API REST que é um estilo de arquitetura para fornecer padrões entre sistemas de computador na Web vai efetuar o controle do banco de dados e vai impor as regras de Inteligência Artificial e Machine Learning. O banco de dados é alimentado pela página na web que consome o serviço API REST que executa a inserção dos dados no banco, gerando assim uma base sólida para o Machine Learning se comunicar com o aplicativo que é um cliente visando o aluno como usuário, assim mediante as requisições dos serviços da API REST, o Machine Learning vai passando a conhecer o aluno em questão, e para cada nova requisição a Inteligência Artificial vai conseguir identificar o grau de dificuldade que o aluno está demonstrando e assim vai calcular o que deve ser respondido para o aplicativo.

O aplicativo vai ser uma biblioteca de jogos (tarefas) com diversas atividades, assim possibilitando a escolha do aluno para qual das atividades the agrada mais. A primeira tarefa que está sendo idealizada é um jogo onde as atividades são mostradas por imagens e definidas com um grau de dificuldade, palavras diversas com somente uma palavra correta para o aluno, dentro desse processo o Machine Learning é implícito, pois a cada ação do usuário a Inteligência Artificial vai aprendendo sobre ele e definindo assim qual vai ser a próxima atividade a ser mostrada.

O projeto tem dois tipos de usuários, um que é quem alimenta o banco de dados, no qual esse seria um profissional capacitado para distinguir os diferentes níveis do autismo e definir níveis de dificuldades para as imagens e palavras a serem usadas nas tarefas, e o outro é o aluno, no qual tem um nível pré-definido de autismo e é quem vai realizar a atividade, assim cada nova atividade gerada pelo sistema é baseada no nível do aluno, nível das imagens e palavras, assim criando uma atividade determinada ao aluno, mostrando uma imagem e sua respectiva palavra correta e três outras palavras definidas também por dificuldade, a missão do aluno é escolher a palavra correta podendo errar ou sair da atividade sem nenhuma penalidade, e para cada tentativa são guardadas suas ações, ou seja, será armazenada a dificuldade das imagens e as palavras que em conjunto somam a dificuldade da tarefa, quantas tentativas, se acertou a palavra e a hora da sua execução. Com esses dados vinculados ao aluno conseguimos via Machine Learning determinar quais atividades o aluno será exposto em seguida, assim gerando um jogo de tarefas onde cada nova atividade é dada com base nas suas ações anteriores. Todos esses dados coletados do aluno podem ser usados pelo profissional capacitado para avaliação do aluno mediante tais questões impostas por ele mesmo ao alimentar o banco.

\section{DISCUSSÃO E RESULTADOS}

A partir da análise dos softwares existentes para as crianças autistas na instituição de ensino e com as entrevistas com pais e professores, observou-se que não há muitos softwares com um grau de adaptabilidade capaz de atender as diversas necessidades que uma criança autista pode apresentar. Diante disso, este trabalho propõe o desenvolvimento de um software que apresente interfaces adaptativas capazes de atender as diferentes necessidades de uma criança autista, além de várias atividades que exploram a curiosidade das crianças pelas diferentes atrações do mundo. Tais atividades os ajudam na capacidade de organizar seus pensamentos com um propósito, promovendo assim o desenvolvimento cognitivo dos mesmos.

Os resultados irão descrever a inspeção que será realizada por profissionais responsáveis pelo tratamento, direcionando a análise aos dados coletados e a inter- 


\section{Computa Rplicada}

face do usuário por questionários. A partir do questionário será possível analisar a experiência do usuário em contato com o software.

É importante mencionar que o software foi desenvolvido visando reforçar a colaboração entre o professor e o aluno, através de um ambiente de jogos para dispositivos móveis cujo objetivo é dar suporte ao tratamento de crianças com autismo. Tal ambiente dispõe-se de uma biblioteca de jogos com diversas categorias e níveis, além de disponibilizar informações pertinentes à jogabilidade da criança tendo como meta de que se possam trabalhar vários conceitos fundamentais para o tratamento. Com a portabilidade para diversas plataformas, o aplicativo tende a ter um custo menor de implantação por clínicas e familiares, uma vez que pode ser embarcado em diferentes dispositivos móveis de baixo custo. Uma vez que o principal objetivo é uma constante melhora da criança autista, trabalhando ainda em suas deficiências específicas através dos dados coletados ao longo dos jogos que poderão servir como métricas para a futura implementação dos sistemas de recomendação de tratamento, ou seja, o software fará um mapeamento discreto do usuário com base nos resultados anteriores a fim de auxiliar a identificação dos diferentes perfis de usuários de maneira automatizada e acompanhar a evolução dos alunos para proporcionar um tratamento de maior qualidade. Por fim, com o objetivo de interagir com um cenário ainda tão desconhecido, como é o universo autista, a pesquisa realizada no desenvolvimento do aplicativo continua de maneira a se aprimorar cada vez mais, bem como as propostas em adicionar outros recursos que auxiliem no tratamento e interação das tecnologias atuais.

\section{CONCLUSÃO}

O autismo é um terreno fértil para investigação, visto que ainda existem tantas perguntas a seu respeito não respondidas. Recentemente, para além dos estudos sobre o universo das tecnologias, a questão dos dispositivos tecnológicos vem recebendo um olhar mais atento quanto às infinitas possibilidades de utilização no setor educacional.

Este tema foi motivado pela necessidade de auxiliar o professor e o aluno, oferecendo um suporte de software de comunicação alternativa para facilitar os processos de aprendizagem e a convivência dos indivíduos com autismo, contribuindo com uma ferramenta para auxiliar a inclusão das crianças autistas, preferencial- mente na rede regular de ensino, já que toda a criança e adolescente têm direito à educação para garantir seu pleno desenvolvimento como pessoa, e prepará-lo para o exercício da cidadania e qualificação para o trabalho visto que estudos nessa área se mostram sempre importantes, que eles proporcionam grandes avanços no que se refere a uma patologia sem cura.

Em razão do desenvolvimento de uma aplicação com inteligência artificial para estímulo e aprendizagem de autistas este artigo apresenta uma proposta de um jogo didático, que simula atividades cotidianas direcionadas a crianças com autismo. Com o objetivo de reforçar e estimular comportamentos, auxiliar na interação social e cognição. O uso do jogo se vê benéfico a partir do momento que inserido na rotina do aluno juntamente a de seu professor, podendo ser aplicado nas instituições de ensino e podendo também ser utilizado paralelamente na sua rotina diária. Além de uma expansão de conhecimento estaremos ultrapassando obstáculos criados pelos preconceitos, falhas de informações e pela própria barreira do transtorno de espectro autista. Atingindo também objetivos pessoais, colocando em prática todos os conhecimentos que vêm sendo desenvolvidos. Satisfazendo assim uma necessidade de levar conhecimento e utilizar a tecnologia em prol daqueles que precisam.

\section{REFERÊNCIAS}

APAE - ASSOCIAÇÃO DE PAIS E AMIGOS DOS EXCEPCIONAIS. Histórico da APAE: 28 anos de história. São Paulo: Federação Nacional das APAES, 2018.

APAE - ASSOCIAÇÃO DE PAIS E AMIGOS DOS EXCEPCIONAIS. Histórico da APAE: 8 anos de história. São Paulo: Federação Nacional das APAES, 2018.

BARTH, C.; PASSERINO, L.; SANTAROSA, L. M. C. Software Descobrindo Emoções: estudo da teoria da mente em autistas. Trabalho apresentado no VII Congresso Ibero-americano de Informática Educativa, 2004. Disponível em: http://www.ufrgs.br/niee/eventos/ RIBIE/2004/comunicacao/com600-609.pdf. Acesso em: 09 jun. 2018.

DAUTENHAHN, K.; BILLARD, A. Games Children with Autism Can Play With Robota, a Humanoid Robotic Doll. Universal Access and Assistive Technology, n. 1, mar. de 2002. 
GALVÃO FILHO, Teófilo. Tecnologia Assistiva nas escolas: recursos básicos de acessibilidade sociodigital para pessoas com deficiência. São Paulo: Instituto de Tecnologia Social (ITS Brasil) - Microsoft | Educação, 2008. Disponível em: https://docs.wixstatic.com/ ugd/85fd89_a9baa902e9c94ce5b8b19e4072baf46a. pdf. Acesso em: 26 maio 2018.

KORTH, Henry F. et al. Sistema de Banco de Dados. 2. ed. Rio de Janeiro: Elsevier, 2004.

LAUDON, K.; LAUDON, J. Sistema de informação Gerenciais. 9. ed. São Paulo: Editora Pearson, 2009.

AMERICAN PSYCHIATRIC ASSOCIATION. DSM-5Manual Diagnóstico e Estatístico de Transtornos Mentais. 5. Ed. São Paulo: Artmed, 2014.

NILSSON, I. A educação de pessoas com desordens do espectro autístico e dificuldades semelhantes de aprendizagem. Temas sobre desenvolvimento, São Paulo, v. 12 , n. 68 , p. 5-45, maio-jun. 2003.

PAIS, Paula. O Autismo e a Aprendizagem escolar. Só Pedagogia. 2013. Disponível em: http://www.pedagogia.com.br/artigos/autismo/. Acesso em: 20 maio 2018.

PASSERINO, L. M.; SANTAROSA, L. M. C.; TAROUCO, L. M. R. Pessoas com Autismo em Ambientes Digitais de Aprendizagem: estudo dos processos de Interação Social e Mediação. Anais... do SBIE 2006.

SALLE, E. et al. Autismo Infantil: Sinais e Sintomas. In: CAMARGOS JR., W. (Org.). Transtornos Invasivos do Desenvolvimento: 3o. Milênio. Brasília: CORDE, 2002. p. 11-15.

SAMUEL, Arthur. "Alguns estudos em aprendizado de máquina usando o jogo de damas". Revista IBM de Pesquisa e Desenvolvimento, v. 3, n. 3 p. 210-229. 